\title{
On some claims in Ramanujan's 'unpublished' manuscript on the partition and tau functions
}

\author{
Pieter Moree
}

\begin{abstract}
We disprove various claims made by Ramanujan in his, until very recently, unpublished manuscript [1] on the partition and tau functions. Furthermore, the second part of a related paper by G.K. Stanley [17] is corrected (the first part of which was earlier corrected by D. Shanks 16]).
\end{abstract}

\section{Introduction}

In his first letter (16 Jan. 1913) to Hardy [2, p. 24], Ramanujan made various claims. The fourth of them reads as follows: "(4) 1,2, 4, 5, 8, 9, 10, 13, 16, 17, 18, $\cdots$ are numbers which are either themselves squares or which can be expressed as the sum of two squares. The number of such numbers greater than $A$ and less than $B$

$$
=K \int_{A}^{B} \frac{d x}{\sqrt{\log x}}+\theta(x)
$$

where $K=0.764 \cdots$ and $\theta(x)$ is very small when compared with the previous integral. $K$ and $\theta(x)$ have been exactly found, though complicated....". (Note that $\theta(x)$ should be $\theta(B)$.) Answering an inquiry of Hardy [Q, p. 49], Ramanujan in his second letter to Hardy [2, p. 56] claims: "The order of $\theta(x)$ which you asked in your letter is $\sqrt{x} / \sqrt{\log x}$ ". In his lectures [6] on Ramanujan's work, Hardy states that Ramanujan also gave the exact value of $K$, namely

$$
K=\frac{1}{\sqrt{2}} \prod_{p \equiv 3(\bmod 4)}\left(1-\frac{1}{p^{2}}\right)^{-1 / 2} .
$$

Note that Ramanujan's claim, if correct, would imply that

$$
B(x)=\frac{K x}{\sqrt{\log x}}\left(1+\frac{C_{2}}{\log x}+O\left(\frac{1}{\log ^{2} x}\right)\right),
$$

P. Moree: KdV Institute, University of Amsterdam, Plantage Muidergracht 24, 1018 TV Amsterdam, The Netherlands, e-mail: moree@science.uva.nl

Mathematics Subject Classification (2001). 01A99, 11N37, 11Y60 
with $C_{2}=1 / 2$, where $B(x)$ denotes the number of integers $n \leq x$ of the form $u^{2}+v^{2}$ with $u$ and $v$ integers. Landau proved in 1908, using contour integration, that asymptotically $B(x) \sim K x / \sqrt{\log x}$. His method can be easily extended [14 to prove that $B(x)$ has an asymptotic expansion in the sense of Poincaré, namely for every integer $r \geq 2$, we have

$$
B(x)=\frac{K x}{\sqrt{\log x}}\left(1+\sum_{j=2}^{r} \frac{C_{j}}{\log ^{j-1} x}+O\left(\frac{1}{\log ^{r} x}\right)\right),
$$

with $C_{2}, \cdots, C_{r}$ constants. Shanks [16], correcting on Stanley [17, computed the second order constant $C_{2}$ to equal $0.5819486 \cdots$ and thus disproved Ramanujan's claim. For an overview of further results in this direction the reader is referred to 10 .

There is some evidence (but see [12, p. 92]) that along with his final letter (12 Jan. 1920) to Hardy, Ramanujan included a manuscript on congruence properties of $\tau(n)$ and $p(n)$. In this manuscript Ramanujan considers, for various special small primes $q$, the quantity $\sum_{n \leq x, q \nmid \tau(n)} 1$ and makes claims similar to (11). He defines $t_{n}=1$ if $\tau(n) \not \equiv 0(\bmod q)$ and $t_{n}=0$ otherwise. He then typically writes: "It is easy to prove by quite elementary methods that $\sum_{k=1}^{n} t_{k}=o(n)$. It can be shown by transcendental methods that

$$
\sum_{k=1}^{n} t_{k} \sim \frac{C n}{(\log n)^{\delta}}
$$

and

$$
\sum_{k=1}^{n} t_{k}=C \int_{1}^{n} \frac{d x}{(\log x)^{\delta}}+O\left(\frac{n}{(\log n)^{r}}\right) .
$$

where $r$ is any positive number". Note that the truth of $\sum_{k=1}^{n} t_{k}=o(n)$ would imply that $q \mid \tau(n)$ for almost all $n$. The values of $\delta$ can be found in the final column of Table 1. Except for $q=5$ and $q=691$ Ramanujan also writes down an Euler product for $C$. It is not difficult to check that these are correct, except when $q=23$, in which case this is due to a factor $\left(1-23^{-s}\right)^{-1}$ erroneously omitted in the generating function.

It appears from [17] that Hardy planned to have this manuscript published under Ramanujan's name after some editing. Indeed, he published some parts of it (see [1]). Unfortunately, he never published Ramanujan's full manuscript (which indeed needed some serious editing). Some of his results in connection with the unpublished manuscript were further worked out by his research student Geraldine Stanley and published in 1928 [17]. She claimed Ramanujan's assertion regarding $C_{2}$ and the second order coefficient for $\sum_{n \leq x, 5 \nmid \tau(n)} 1$ to be false. Unfortunately, her paper contains several typos and some mathematical errors, which were corrected by Shanks [16] for the $B(x)$ case and are corrected in this paper for the $5 \mid \tau(n)$ case in Section 10.

In 1928 Hardy passed on his Ramanujan materials to G.N. Watson. Watson's papers [20, 21, 22] are inspired by Ramanujan's 'unpublished' manuscript. In particular Watson [20] rigorously proved Ramanujan's assertion that $691 \mid \tau(n)$ for almost all $n$ by establishing (2) with $q=691$ and $\delta=1 / 690$. A remarkable 
result, since for $1 \leq n \leq 5000$ we have, as Ramanujan computed, $691 \nmid \tau(n)$, except for the multiples of 1381 in that range. After Watson's death in 1965 a part of the manuscript came into the possession of the library of Trinity College, Cambridge. Watson's copy of the remaining part can be found in the library of Oxford's Mathematical Institute. A full version of the unpublished manuscript (with proofs and commentary) has only recently become available to the general public [1].

Ramanujan's claims are only the tip of an iceberg: it is now known [14, 15] that if $\sum_{n=1}^{\infty} a_{n} q^{n}$, with $q=e^{2 \pi i z}$, is the Fourier expansion of a modular form of integral weight with integral coefficients for a congruence subgroup of the full modular group, then for every positive integer $M$, for almost all $n$ we have $a(n) \equiv 0(\bmod M)$. In particular, we have that (2) holds true for every odd prime $q$ not in Table 1 with $\delta=q /\left(q^{2}-1\right)$. It can also be shown [18], using $l$-adic representations, that 2 and the primes in Table 1 are the only primes for which congruences for $\tau(n)$ exist.

The purpose of this paper is to establish the falsity of the various claims made by Ramanujan of the type (3). We do this by evaluating the relevant second order coefficients and compute them with at least a few decimals of precision. Moreover, we will correct the part of Stanley's paper pertaining to the $5 \mid \tau(n)$ case. As far as possible we adopt Ramanujan's notation and the order in which we deal with the various primes follows the order in which they appear in Ramanujan's manuscript.

\section{Some relevant general results}

In this section we quote some general results that allow us to evaluate the secondorder constants of the functions considered by Ramanujan.

The following result was obtained by Moree 9] using elementary methods. It can be deduced on analysing an appropriate functional equation (apparently first considered by Levin and Fainleib) for the relevant counting function. Given a multiplicative function $f$, we define $\Lambda_{f}(n)$ implicitly by

$$
f(n) \log n=\sum_{d \mid n} f(d) \Lambda_{f}\left(\frac{n}{d}\right) .
$$

We denote the formal Dirichlet series $\sum_{n=1}^{\infty} f(n) n^{-s}$ associated to $f$ by $L_{f}(s)$. It is easy to see that $-L_{f}^{\prime}(s) / L_{f}(s)=\sum_{n=1}^{\infty} \Lambda_{f}(n) n^{-s}$. As usual the logarithmic integral $\int_{2}^{x} d t / \log t$ is denoted by $\operatorname{Li}(x)$.

Theorem 1 [9]. Let $f$ be a multiplicative function satisfying

$$
0 \leq f\left(p^{r}\right) \leq c_{1} c_{2}^{r}, c_{1} \geq 1,1 \leq c_{2}<2,
$$

and $\sum_{p \leq x} f(p)=\tau \operatorname{Li}(x)+O\left(x \log ^{-2-\rho} x\right)$, where $\tau$ and $\rho>1$ are positive real fixed numbers. Then, for some $\epsilon>0$ and constant $B_{f}$ we have

$$
\sum_{n \leq x} \frac{\Lambda_{f}(n)}{n}=\tau \log x+B_{f}+O\left(\frac{x}{\log ^{1+\epsilon} x}\right) .
$$


There exist constants $\epsilon^{\prime}>0$ and constants $C_{1}(f)>0$ and $C_{2}(f)$ such that

$$
\sum_{n \leq x} f(n)=\frac{C_{1}(f) x}{\log ^{1-\tau} x}\left(1+\frac{C_{2}(f)}{\log x}+O\left(\frac{1}{\log ^{1+\epsilon^{\prime}} x}\right)\right), x \rightarrow \infty .
$$

In particular, $C_{2}(f)=(1-\tau)\left(1+B_{f}\right)$.

Remark. Alternatively we can write

$$
C_{2}(f)=(1-\tau)\left(1+\tau \gamma+\sum_{n=1}^{\infty} \frac{\Lambda_{f}(n)-\tau}{n}\right)
$$

where $\gamma$ denotes Euler's constant.

Corollary 1 Let $f$ satisfy the conditions of Theorem 1 with $\tau \neq 1$. Suppose that

$$
\sum_{n \leq x} f(n)=C_{1}(f) \int_{1}^{x} \frac{d t}{\log ^{1-\tau} t}+O\left(\frac{x}{\log ^{r} x}\right)
$$

for some $r>2-\tau$, then we must have $B_{f}=0$.

The constant $B_{f}$ appearing in (5) can be computed using the following result.

Lemma 1 Let $f$ satisfy the conditions of Theorem 1 and let $B_{f}$ and $\tau$ be defined as in Theorem 1. Then

$$
B_{f}=-\lim _{s \rightarrow 1+0}\left(\frac{L_{f}^{\prime}(s)}{L_{f}(s)}+\frac{\tau}{s-1}\right) .
$$

Example. Take $f=\mathbf{1}$, that is $f(n)=1$ for every $n \geq 1$. Then $\Lambda_{f}$ equals the Von Mangoldt function and $L_{f}(s)=\zeta(s)$. Using the well-known Taylor expansion

$$
\zeta(s)=\frac{1}{s-1}+\gamma+o(1)
$$

around $s=1$, we obtain that $B_{f}=-\gamma$.

Our proof of Lemma 1] will make use of the following result due to Landau 8, pp. 73-74].

Lemma 2 Suppose that $\sum_{n \leq x} h(n)=\alpha x+O(g(x))$, where $g(x)$ is a positive function of $x$ such that $g(x) / x^{2}$ is monotonically decreasing for every $x \geq x_{0}, x_{0}$ some fixed number, and where $\int_{x_{0}}^{\infty} g(t) d t / t^{2}<\infty$. Then, for $\operatorname{Re}(s)>1$, we have the estimate

$$
\sum_{n=1}^{\infty} \frac{h(n)}{n^{s}}=\frac{\alpha}{s-1}+\beta+o(1), s \rightarrow 1+0,
$$

for some constant $\beta$. Furthermore, we have

$$
\sum_{n \leq x} \frac{h(n)}{n}=\alpha \log x+\beta+o(1), x \rightarrow \infty .
$$


We are now in the position to prove Lemma 1.

Proof of Lemma 1. By partial integration we deduce from (5) that $\sum_{n \leq x} \Lambda_{f}(n)=$ $\tau x+O\left(x \log ^{-1-\epsilon} x\right)$. We thus can invoke Lemma 2 with $h(n)=\Lambda_{f}(n), \alpha=\tau$ and $g(x)=x \log ^{-1-\epsilon} x$. On noting that $-L_{f}^{\prime}(s) / L_{f}(s)=\sum_{n=1}^{\infty} \Lambda_{f}(n) n^{-s}$, the result then follows.

Serre [14 gave some beautiful applications of Landau's method to counting functions involving coefficients of modular forms. In order to formulate his result we have to define the concept of Frobenius density. A set of primes $\mathcal{P}$ is called Frobenius of density $\delta$, if there exists a finite Galois extension $K / \mathbb{Q}$ and a subset $H$ of $G:=\operatorname{Gal}(K / \mathbb{Q})$ such that $H$ is stable under conjugation, $|H| /|G|=\delta$ and for every prime $p$, with at most finitely many exceptions, one has $p \in \mathcal{P}$ if and only if $\sigma_{p}(K / \mathbb{Q}) \in H$, where $\sigma_{p}(K / \mathbb{Q})$ denotes the Frobenius map of $p$ in $G$ (defined modulo conjugation in case $p$ does not divide the discriminant of $K$ ). Serre [14, using's Landau's contour integration method, established the following result, which we formulate here in a slightly less general form.

Theorem 2 [14, Théorème 2.8]. Let $\alpha(n): \mathbb{N} \rightarrow \mathbb{Z}$ be a multiplicative function. Let $q$ be a fixed prime. Suppose that the set $P:=\{p$ is prime : $q \mid \alpha(p)\}$ is Frobenian of density $0<1-\tau<1$. Let $h(s)=\sum_{q \nmid \alpha(n)} n^{-s}$. Then around $s=1$ we have

$$
\frac{h(s)}{s}=\frac{1}{(s-1)^{\tau}}\left(e_{0}+e_{1}(s-1)+\cdots+e_{k}(s-1)^{k}+\cdots\right),
$$

for certain numbers $e_{j}$ with $e_{0} \neq 0$ and we have, furthermore,

$$
\sum_{\substack{n \leq x \\ q \nmid \alpha(n)}} 1=\frac{e_{0} x}{\Gamma(\tau) \log ^{1-\tau} x}\left(1+\cdots+\frac{\Gamma(\tau) e_{k}}{\Gamma(\tau-k) e_{0} \log ^{k} x}+O\left(\frac{1}{\log ^{k+1} x}\right)\right) .
$$

In particular the second order constant, $\Gamma(\tau) e_{1} /\left(\Gamma(\tau-1) e_{0}\right)$, equals

$$
(1-\tau) \lim _{s \rightarrow 1+0}\left(-\frac{L_{f}^{\prime}(s)}{L_{f}(s)}-\frac{\tau}{s-1}+1\right) .
$$

It can be shown that if the conditions of Theorem 2 are satisfied, also the conditions of Theorem 1 are satisfied. Theorem 1 in combination with Lemma 1 and Theorem 2 both predict the same second order coefficient.

Let $T(s)=\sum_{n=1}^{\infty} t_{n} / n^{s}$. The approach followed in disproving Ramanujan's claims of the format (3) is to write $T(s)=\zeta(s)^{\tau} g(s)$ with $g(s)$ a regular function for $\operatorname{Re}(s)>1 / 2$. By Lemma 1 and (6) it then follows that $B_{t}=-\tau \gamma-g^{\prime}(1) / g(1)$. We have $\tau=1-\delta$. The numerical work (carried out in Section 9) shows that $B_{t} \neq 0$. From Corollary 1 the falsity of Ramanujan's claim then follows for every $r>2-\tau$.

\section{Divisibility of tau by 2}

Ramanujan shows that $\tau(n)$ is odd or even according as $n$ is an odd square or not. It thus follows that $\sum_{n \leq x, 2 \nmid \tau(n)} 1=\left[\frac{1+\sqrt{x}}{2}\right]$. 


\section{Divisibility of tau by 5}

At [1, p. 47] Ramanujan makes a statement of the form (3) with $q=5$ and $\delta=1 / 4$. Put $t_{n}=0$ if $5 \mid \tau(n)$ and $t_{n}=1$ otherwise. Let $T(s)=\sum_{n=1}^{\infty} t_{n} / n^{s}$. Denote $\sum_{d \mid n} d^{r}$ by $\sigma_{r}(n)$. On using that $\tau(n) \equiv n \sigma_{1}(n)(\bmod 5)$, it is easily seen that $T(s)$ equals

$$
\prod_{p \equiv 1(\bmod 5)} \frac{1-p^{-4 s}}{\left(1-p^{-s}\right)\left(1-p^{-5 s}\right)} \prod_{p \equiv \pm 2(\bmod 5)} \frac{1-p^{-3 s}}{\left(1-p^{-s}\right)\left(1-p^{-4 s}\right)} \prod_{p \equiv 4(\bmod 5)} \frac{1}{1-p^{-2 s}} .
$$

Let $\chi_{c}$ be the character of $(\mathbb{Z} / 5 \mathbb{Z})^{*}$ that is determined by $\chi_{c}(\overline{2})=i$ and $\chi_{5}$ be the character that is determined by $\chi_{5}(\overline{2})=-1$. Denote

$$
\prod_{p \equiv 1(\bmod 5)}\left(\frac{1-p^{-4 s}}{1-p^{-5 s}}\right)^{4} \prod_{p \equiv \pm 2(\bmod 5)} \frac{\left(1-p^{-3 s}\right)^{4}}{\left(1-p^{-2 s}\right)^{2}\left(1-p^{-4 s}\right)^{3}} \prod_{p \equiv 4(\bmod 5)}\left(1-p^{-2 s}\right)^{-2}
$$

by $H(s)$. Note that

$$
T(s)^{4}=\left(1-5^{-s}\right)^{3} H(s) \zeta(s)^{3} \frac{L\left(s, \chi_{c}\right) L\left(s, \bar{\chi}_{c}\right)}{L\left(s, \chi_{5}\right)} .
$$

Put

$$
D=\prod_{p \equiv 1(\bmod 5)} \frac{1-p^{-4}}{1-p^{-5}} \prod_{p \equiv \pm 2(\bmod 5)} \frac{1-p^{-3}}{\left(1-p^{-2}\right)^{1 / 2}\left(1-p^{-4}\right)^{3 / 4}} \prod_{p \equiv 4(\bmod 5)} \frac{1}{\sqrt{1-p^{-2}}}
$$

Thus the first order Landau-Ramanujan constant $C$ in this case, which was not written down by Ramanujan, equals

$$
C=\frac{1}{\Gamma\left(\frac{3}{4}\right)}\left(\frac{64 L\left(1, \chi_{c}\right) L\left(1, \bar{\chi}_{c}\right)}{125 L\left(1, \chi_{5}\right)}\right)^{1 / 4} D
$$

At [5, p. 388] the $L$-values above are given (for an excellent discussion of how to compute these values see [5, 6.5]); we have

$$
L\left(1, \chi_{c}\right)=\overline{L\left(1, \bar{\chi}_{c}\right)}=\frac{2 \pi}{25}(3-i)\left(\sin \left(\frac{2 \pi}{5}\right)+i \sin \left(\frac{4 \pi}{5}\right)\right)
$$

and $L\left(1, \chi_{5}\right)=\log \left(\frac{3+\sqrt{5}}{2}\right) / \sqrt{5}$. On using that $\sin ^{2}(2 \pi / 5)=(5+\sqrt{5}) / 8$ and $\sin ^{2}(4 \pi / 5)=(5-\sqrt{5}) / 8$, we deduce that $L\left(1, \chi_{c}\right) L\left(1, \bar{\chi}_{c}\right)=2 \pi^{2} / 25$. Alternatively we may deduce the latter equality by noting that

$$
\frac{h(K) 2^{r_{1}}(2 \pi)^{r_{2}} R(K)}{\sqrt{|d(K)|} w(K)}=\operatorname{Res}_{s=1} \zeta_{\mathbb{Q}\left(\zeta_{5}\right)}(s)=L\left(1, \chi_{c}\right) L\left(1, \bar{\chi}_{c}\right) L\left(1, \chi_{5}\right),
$$

where $\zeta_{\mathbb{Q}\left(\zeta_{5}\right)}(s)$ denotes the Dedekind zeta-function of the cyclotomic field $K:=$ $\mathbb{Q}\left(\zeta_{5}\right)$. It is not difficult to show that $h(K)=1, r_{1}=0, r_{2}=2, R(K)=$ 
$\log ((3+\sqrt{5}) / 2), d(K)=125$ and $w(K)=10$ (all of this can be deduced from results proved e.g. in [19]). We thus obtain that

$$
C=\frac{4}{5 \Gamma\left(\frac{3}{4}\right)}\left(\frac{\pi^{2}}{2 \sqrt{5} \log \left(\frac{3+\sqrt{5}}{2}\right)}\right)^{1 / 4} D
$$

Using the prime number theorem for arithmetic progressions we see that the conditions of Lemma 11 are satisfied and from (7) we deduce that

$$
4 B_{t}=-3 \gamma-2 \Re\left(\frac{L^{\prime}\left(1, \chi_{c}\right)}{L\left(1, \chi_{c}\right)}\right)+\frac{L^{\prime}\left(1, \chi_{5}\right)}{L\left(1, \chi_{5}\right)}-\frac{3}{4} \log 5+A_{ \pm 1}+A_{ \pm 2} .
$$

with

$$
\begin{gathered}
A_{ \pm 1}=\sum_{p \equiv 1(\bmod 5)} \log p\left(\frac{-16}{p^{4}-1}+\frac{20}{p^{5}-1}\right)+4 \sum_{p \equiv 4(\bmod 5)} \frac{\log p}{p^{2}-1} \text { and } \\
A_{ \pm 2}=\sum_{p \equiv \pm 2(\bmod 5)} \log p\left(\frac{4}{p^{2}-1}-\frac{12}{p^{3}-1}+\frac{12}{p^{4}-1}\right)
\end{gathered}
$$

\section{$5 \quad$ Divisibility of tau by 7}

At [1, p. 52] Ramanujan makes a statement of the form (3) with $q=7$ and $\delta=$ $1 / 2$. Put $t_{n}=0$ if $7 \mid \tau(n)$ and $t_{n}=1$ otherwise. Using that $\tau(n) \equiv n \sigma_{3}(n)(\bmod 7)$ it is easily seen that

$$
T(s)=\prod_{p \equiv 3,5,6(\bmod 7)} \frac{1}{1-p^{-2 s}} \prod_{p \equiv 1,2,4(\bmod 7)} \frac{1-p^{-6 s}}{\left(1-p^{-s}\right)\left(1-p^{-7 s}\right)} .
$$

A simple compuation shows that

$$
T(s)^{2}=\zeta(s) L\left(s, \chi_{-7}\right)\left(1-7^{-s}\right) \prod_{p \equiv 3,5,6(\bmod 7)} \frac{1}{1-p^{-2 s}} \prod_{p \equiv 1,2,4(\bmod 7)}\left(\frac{1-p^{-6 s}}{1-p^{-7 s}}\right)^{2}
$$

where $\chi_{-7}$ denotes the usual Kronecker character of the number field $\mathbb{Q}(\sqrt{-7})$. From this relation we then obtain

$$
\begin{aligned}
2 B_{t}= & -\gamma-\frac{\log 7}{6}-\frac{L^{\prime}\left(1, \chi_{-7}\right)}{L\left(1, \chi_{-7}\right)}+2 \sum_{p \equiv 3,5,6(\bmod 7)} \frac{\log p}{p^{2}-1} \\
& +\sum_{p \equiv 1,2,4(\bmod 7)} \log p\left(\frac{14}{p^{7}-1}-\frac{12}{p^{6}-1}\right) .
\end{aligned}
$$




\section{Divisibility of tau and lambda by 3}

At [1], p. 64] Ramanujan makes two statements of the form (3) with $q=3$ and $\delta=1 / 2$. Put $t_{n}=0$ if $3 \mid \tau(n)$ and $t_{n}=1$ otherwise. Using that $\tau(n) \equiv$ $n \sigma_{1}(n)(\bmod 3)$, where $\sigma_{1}(n)$ denotes the sum of the positive divisors of $n$, it is easy to see that $t_{n}$ is multiplicative and that

$$
T(s):=\sum_{n=1}^{\infty} \frac{t(n)}{n^{s}}=\prod_{p \equiv 2(\bmod 3)} \frac{1}{1-p^{-2 s}} \prod_{p \equiv 1(\bmod 3)} \frac{1+p^{-s}}{1-p^{-3 s}} .
$$

A simple compuation shows that

$$
T(s)^{2}=\zeta(s) L\left(s, \chi_{-3}\right)\left(1-3^{-s}\right) \prod_{p \equiv 2(\bmod 3)} \frac{1}{1-p^{-2 s}} \prod_{p \equiv 1(\bmod 3)}\left(\frac{1-p^{-2 s}}{1-p^{-3 s}}\right)^{2}
$$

where $\chi_{-3}$ denotes the Kronecker character of the number field $\mathbb{Q}(\sqrt{-3})$. From this relation we then obtain

$$
\begin{aligned}
2 B_{t}= & -\gamma-\frac{\log 3}{2}-\frac{L^{\prime}\left(1, \chi_{-3}\right)}{L\left(1, \chi_{-3}\right)}+2 \sum_{p \equiv 2(\bmod 3)} \frac{\log p}{p^{2}-1} \\
& +\sum_{p \equiv 1(\bmod 3)} \log p\left(\frac{6}{p^{3}-1}-\frac{4}{p^{2}-1}\right) .
\end{aligned}
$$

Let $\lambda(n)$ denote the number of partitions of $n$ as the sum of integers which are not multiples of 9 . Put $l_{n}=0$ if $3 \mid \lambda(n)$ and $l_{n}=1$ otherwise. Ramanujan shows that

$$
\sum_{k \leq x} l_{k}=\sum_{k \leq 3 x+1} t_{k}
$$

and then states, [1, (11.8a)], that it can be shown by transcendental methods that

$$
\sum_{k=1}^{n} l_{k}=C \int_{1}^{n} \frac{d x}{(\log x)^{1 / 2}}+O\left(\frac{n}{(\log n)^{r}}\right)
$$

and

$$
\sum_{k=1}^{n} t_{k}=\frac{C}{3} \int_{1}^{n} \frac{d x}{(\log x)^{1 / 2}}+O\left(\frac{n}{(\log n)^{r}}\right)
$$

where he gives an explicit expression for $C$. Though these claims are correct for $r \leq 3 / 2$, a priori they cannot be both true for $r>3 / 2$, as we then trivially have from (9) that $C_{2}(l)=C_{2}(t)-\frac{1}{2} \log 3$, whereas the truth of both claims of Ramanujan would imply that $C_{2}(l)=C_{2}(t)$.

\section{Divisibility of tau by 691}

At [1], p. 66] Ramanujan makes a statement of the form (3) with $q=691$ and $\delta=1 / 690$. He did not write down an explicit first order constant. The truth of this assertion for $r \leq 691 / 690$ was first established by G.N. Watson 20]. In this 
note it will be shown, however, that the statement is false for every $r>691 / 690$.

It is not difficult to show, as Ramanujan did, that $\tau(n) \equiv \sigma_{11}(n)(\bmod 691)$. Let $\nu(p)$ be the smallest integer $>1$, such that $p^{\nu(p)} \equiv 1(\bmod 691)$. We put $\nu(691)=\infty$. Note that $\sigma_{11}\left(p^{k}\right) \equiv 0(\bmod 691)$ if and only if $k \equiv \nu(p)-$ $1(\bmod \nu(p))$. In case $p=691$ we interpret this congruence as never being satisfied and $1-691^{-\nu(691) s}$ as being 1 . We thus can write

$$
T(s)=\prod_{p} \frac{1-p^{-(\nu(p)-1) s}}{\left(1-p^{-s}\right)\left(1-p^{-\nu(p) s}\right)} .
$$

Around $s=1$ this function is quite close to $\zeta(s)$ (we have $T(s)=\sum_{n=1}^{11053} n^{-s}-$ $\left.\sum_{m=1}^{8}(m 1381)^{-s}-5527^{-s}-8291^{-s}+\sum_{n=11054}^{\infty} t_{n} / n^{s}\right)$ and hence we expect $B_{t}$ to be close to $-\gamma$, cf. Example 1, which is indeed the case by Table 1 .

Notice that each local factor of $T(s)$ has the term $1-p^{-s}$ in it, unless $p \equiv$ $-1(\bmod 691)$, in which case the local factor is $\left(1-p^{-2 s}\right)^{-1}$. By multiplying $T(s)$ with $\prod_{p \equiv-1(\bmod 691)}\left(1-p^{-s}\right)^{-1}$ we can then write it as $\zeta(s) h(s)$ with $h(s)$ a regular function for $\operatorname{Re}(s)>1 / 2$. On noting that the primes $p$ with $p \equiv-1(\bmod 691)$ are exactly the primes that split completely in $\mathbb{Q}\left(\zeta_{691}\right)$ but not in $\mathbb{Q}\left(\cos \left(\frac{2 \pi}{691}\right)\right)$, the product $\prod_{p \equiv-1(\bmod 691)}\left(1-p^{-s}\right)^{-1}$ can be expressed in terms of the Dedekind zetafunctions of the latter two fields and some regular function for $\operatorname{Re}(s)>1 / 2$, which can be explicitly determined using the splitting behaviour of a prime $p$ in these fields. Using the factorisation of the these Dedekind zetafunctions in terms of L-series, we then obtain the following identity, with $\chi_{c}$ the character uniquely determined by $\chi_{c}(\overline{3})=\exp (2 \pi i / 690)$;

$$
\begin{aligned}
& T(s)^{690}=\zeta(s)^{689}\left(1-691^{-s}\right)^{-1} L\left(s, \chi_{c}\right) \prod_{j=1}^{344} \frac{L\left(s, \chi_{c}^{2 j+1}\right)}{L\left(s, \chi_{c}^{2 j}\right)} \\
& \prod_{p \equiv-1(\bmod 691)}\left(1-p^{-2 s}\right)^{-345} \prod_{p \equiv 1(\bmod 691)}\left(\frac{1-p^{-690 s}}{1-p^{-691 s}}\right)^{690} \\
& \prod_{\substack{p \neq 691 \\
2 \mid \nu(p), \nu(p) \geq 4}}\left(\frac{1+p^{-\nu(p) \frac{s}{2}}}{1-p^{-\nu(p) \frac{s}{2}}}\right)^{\frac{690}{\nu(p)}} \prod_{\substack{p \\
2<\nu(p)<691}}\left(\frac{1-p^{-(\nu(p)-1) s}}{1-p^{-\nu(p) s}}\right)^{690},
\end{aligned}
$$

the truth of which is most easily established by checking that the local factors on both sides agree for every prime $p$. As before a formula for $B_{t}$ can now be easily written down, but for reasons of space we leave this to the interested reader. It turns out that the contribution of the last four products in the formula for $T(s)^{690}$ to $B_{t}$ is less than $10^{-5}$ in absolute value. We thus have

$$
B_{t} \approx \frac{\log 691}{690^{2}}-\frac{689}{690} \gamma-\frac{1}{690} \sum_{j=0}^{344} \frac{L^{\prime}\left(1, \chi_{c}^{2 j+1}\right)}{L\left(1, \chi_{c}^{2 j+1}\right)}+\frac{1}{690} \sum_{j=1}^{344} \frac{L^{\prime}\left(1, \chi_{c}^{2 j}\right)}{L\left(1, \chi_{c}^{2 j}\right)} .
$$

with an error of at most $10^{-5}$.

\section{Divisibility of tau by 23}

At [1, p. 80] Ramanujan makes a statement of the form (3) with $q=23$ and $\delta=1 / 2$. Using a trick of Wilton [24], $T(s)$ can be found in this case more easily 
than by Ramanujan's approach. Using Euler's identity we note that

$$
\begin{aligned}
\sum_{n=1}^{\infty} \tau(n) x^{n} & =x\left\{(1-x)\left(1-x^{2}\right) \cdots\right\}^{23}(1-x)\left(1-x^{2}\right) \cdots \\
& \equiv x k\left(x^{23}\right) \sum_{m=-\infty}^{\infty}(-1)^{m} x^{\frac{m(3 m+1)}{2}}(\bmod 23),
\end{aligned}
$$

where $k(x)=\prod_{n=1}^{\infty}\left(1-x^{n}\right)$. Now if we also apply Euler's identity to $k\left(x^{23}\right)$, then we obtain

$$
\sum_{n=1}^{\infty} \tau(n) x^{n} \equiv x \sum_{r=-\infty}^{\infty} \sum_{m=-\infty}^{\infty}(-1)^{m+r} x^{\frac{m(3 m+1)}{2}+23 \frac{r(r+1)}{2}}(\bmod 23) .
$$

From the latter identity Wilton's congruences are easily deduced;

$$
\tau(p) \equiv\left\{\begin{array}{ll}
1(\bmod 23) & \text { if } p=23 \\
0(\bmod 23) & \text { if }(p / 23)=-1 \\
2(\bmod 23) & \text { if } p=U^{2}+23 V^{2} \\
-1(\bmod 23) & \text { for other } p \neq 23
\end{array} \text { with } U \neq 0 ;\right.
$$

Using that $\tau\left(p^{k+1}\right)=\tau(p) \tau\left(p^{k}\right)-p^{11} \tau\left(p^{k-1}\right)$ for $k \geq 1$, we can now easily compute $\tau\left(p^{k}\right)$ modulo 23. Let $\mathcal{S}_{1}$ denote the set of primes $p$ such that $p$ is a quadratic non-residue $\bmod 23$. Let $\mathcal{S}_{3}$ denote the set of primes $p$ which can be written as $U^{2}+23 V^{2}$ with $U \neq 0$. Let $\mathcal{S}_{2}$ be the set of remaining primes $p \neq 23$. (The sets $\mathcal{S}_{1}, \mathcal{S}_{2}, \mathcal{S}_{3}$ have natural densities of respectively, $1 / 2,1 / 3$ and $1 / 6$, which can be shown using that the class number of $\mathbb{Q}(\sqrt{-23})$ equals 3.) We now find that

$$
T(s)=\frac{1}{1-23^{-s}} \prod_{p \in \mathcal{S}_{1}} \frac{1}{1-p^{-2 s}} \prod_{p \in \mathcal{S}_{2}} \frac{1+p^{-s}}{1-p^{-3 s}} \prod_{p \in \mathcal{S}_{3}} \frac{1-p^{-22 s}}{\left(1-p^{-s}\right)\left(1-p^{-23 s}\right)} .
$$

The factor at 23 is not present in Ramanujan's formula (17.6), although it should be there according to his argument. This leads then to an incorrect formula for the first order constant (at the bottom of [1, p. 80]). On should replace the factor $66^{1 / 2} 23^{-3 / 4}$ by $23^{1 / 4} \sqrt{3 / 22}$. It is also clear that where Ramanujan writes 'all primes of the form $23 a^{2}+b^{2}$, he excludes the prime 23 .

We have

$$
T(s)^{2}=\frac{\zeta(s) L\left(s, \chi_{-23}\right)}{1-23^{-s}} \prod_{p \in \mathcal{S}_{1}} \frac{1}{1-p^{-2 s}} \prod_{p \in \mathcal{S}_{2}}\left(\frac{1-p^{-2 s}}{1-p^{-3 s}}\right)^{2} \prod_{p \in \mathcal{S}_{3}}\left(\frac{1-p^{-22 s}}{1-p^{-23 s}}\right)^{2} .
$$

From this we easily deduce that

$$
\begin{aligned}
B_{t}= & -\frac{\gamma}{2}-\frac{L^{\prime}\left(1, \chi_{-23}\right)}{2 L\left(1, \chi_{-23}\right)}+\frac{\log 23}{44} \\
& +\sum_{p^{11} \equiv-1(\bmod 23)} \frac{\log p}{p^{2}-1}+\sum_{p^{11} \equiv 1(\bmod 23)} \log p\left(\frac{3}{p^{3}-1}-\frac{2}{p^{2}-1}\right) \\
& +\sum_{\substack{p=U^{2}+23 V^{2} \\
p>23}} \log p\left(\frac{2}{p^{2}-1}-\frac{3}{p^{3}-1}+\frac{23}{p^{23}-1}-\frac{22}{p^{22}-1}\right) .
\end{aligned}
$$

Remark. To the reader familiar with Cox's beautiful book [3, we suggest as an exercise showing that $p \in \mathcal{S}_{3}$ if and only if $\left(\begin{array}{c}p \\ 23\end{array}\right)=1$ and the congruence $x^{3} \equiv x+1(\bmod p)$ has an integer solution. 


\section{Numerical evaluation of the second order con- stants}

The expressions obtained for the various $B_{t}$ involve both prime sums and values of $L$ and $L^{\prime}$ at $s=1$. The prime sums we evaluate termwise and estimate the tail using that, for $k>1$ and $x \geq 7481$,

$$
\sum_{p>x} \frac{\log p}{p^{k}-1} \leq \frac{x}{x^{k}-1}\left(-0.98+1.017 \frac{k}{k-1}\right)
$$

which follows easily on using the estimate $0.98 x \leq \theta(x) \leq 1.017 x$ for $x \geq 7481$ [13.

The $L$ and $L^{\prime}$ values above can be evaluated using generalized Euler constants for arithmetical progressions. We define

$$
\gamma_{k}(r, m):=\lim _{x \rightarrow \infty}\left\{\sum_{\substack{0<n \leq x \\ n \equiv r(\bmod m)}} \frac{\log ^{k} n}{n}-\frac{\log ^{k+1} x}{m(k+1)}\right\} .
$$

Note that $\gamma_{0}(0,1)=\gamma$, Euler's constant. Let $\chi$ be a non-principal character modulo $m$. It is not difficult to show [7] that for $k \geq 0$ we have

$$
L^{(k)}(1, \chi)=(-1)^{k} \sum_{r=1}^{m} \chi(r) \gamma_{k}(r, m) \text {. }
$$

Using Proposition 12 of [4], the Euler constants $\gamma_{k}(r, m)$ can be computed with any degree of precision and thus the same holds true for $L^{(k)}(1, \chi)$.

For $q=5$ we find, using Dilcher's Table 1 [4, S21], that $L^{\prime}\left(1, \chi_{5}\right) / L\left(1, \chi_{5}\right)=$ $0.82767947 \cdots$ and $L^{\prime}\left(1, \chi_{c}\right) / L\left(1, \chi_{c}\right)=0.15786453 \cdots-i 0.08833613 \cdots$. For $q=7$ we find using Dirichlet's formula that $L\left(1, \chi_{-7}\right)=\pi / \sqrt{7}$ and, using Dilcher's Table 1 , that $L^{\prime}\left(1, \chi_{-7}\right)=0.01856598 \cdots$. The quotient $L^{\prime}\left(1, \chi_{-3}\right) / L\left(1, \chi_{-3}\right)$ is evaluated with many decimal accuracy in, e.g., [9]. We have $L\left(1, \chi_{-23}\right)=3 \pi / \sqrt{23}$ and, on implementing Proposition 12 of [4] in Maple, we find $L^{\prime}\left(1, \chi_{-23}\right)=$ $-0.82955295 \cdots$. Similarly we find that the sum involving the odd, respectively even characters in (10) equal $1.9018228 \cdots$, respectively $5.10942407 \cdots$ (note that a priori these sums must be real).

For $q=3$ we can use the relationship $-\zeta^{\prime}(2) / \zeta(2)=\sum_{p} \log p /\left(p^{2}-1\right)$ to rewrite $B_{t}$ as

$$
2 B_{t}=6 \sum_{p \equiv 2(\bmod 3)} \frac{\log p}{p^{2}-1}+4 \frac{\zeta^{\prime}(2)}{\zeta(2)}-\frac{L^{\prime}\left(1, \chi_{-3}\right)}{L\left(1, \chi_{-3}\right)}-\gamma+6 \sum_{p \equiv 1(\bmod 3)} \frac{\log p}{p^{3}-1} .
$$

Since all but the last term were either computed with high accuracy in [9] or are easily computable with high accuracy in Maple, we now obtain that $B_{t}=-0.5349219 \cdots$.

Let us for a function $f$ satisfying the conditions of Theorem 1 define $H_{f}(x):=$ $\sum_{n \leq x} \Lambda_{f}(n) / n-\tau \log x$. The function $\Lambda_{f}$ is most easily computed by computing minus the logarithm of the generating series of $f$. The numbers $H_{f}\left(10^{5}\right)$ and 
$H_{f}\left(10^{6}\right)$ ought to be approximations of $B_{f}$. The function $b$ is the indicator function of the set of integers that can be written as a sum of two squares. This is, as was already known to Fermat, a multiplicative function. The final column in Table 1 gives Ramanujan's predicted value for $C_{2}$.

Table 1: Numerical values

\begin{tabular}{|c|c|c|c|c|c|c|}
\hline & $f$ & $H_{f}\left(10^{5}\right)$ & $H_{f}\left(10^{6}\right)$ & $B_{f}$ & $C_{2}(f)$ & R's $C_{2}$ \\
\hline \hline$B(x)$ & $\mathrm{b}$ & $+0.163 \cdots$ & $+0.162 \cdots$ & $+0.1638 \cdots$ & $0.5819 \cdots$ & $1 / 2$ \\
\hline$q=5$ & $\mathrm{t}$ & $-0.401 \cdots$ & $-0.400 \cdots$ & $-0.3995 \cdots$ & $0.1501 \cdots$ & $1 / 4$ \\
\hline$q=7$ & $\mathrm{t}$ & $-0.232 \cdots$ & $-0.232 \cdots$ & $-0.2316 \cdots$ & $0.3841 \cdots$ & $1 / 2$ \\
\hline$q=3$ & $\mathrm{t}$ & $-0.532 \cdots$ & $-0.534 \cdots$ & $-0.5349 \cdots$ & $0.2325 \cdots$ & $1 / 2$ \\
\hline$q=691$ & $\mathrm{t}$ & $-0.571 \cdots$ & $-0.571 \cdots$ & $-0.5717 \cdots$ & $0.0006 \cdots$ & $1 / 690$ \\
\hline$q=23$ & $\mathrm{t}$ & $-0.217 \cdots$ & $-0.217 \cdots$ & $-0.2166 \cdots$ & $0.6083 \cdots$ & $1 / 2$ \\
\hline
\end{tabular}

Remark. The computations were not carried out far enough to determine the fifth digit in $-0.2166 \cdots$; it is either a 6 or a 7 .

Now we are in the position to prove the following result.

Theorem 3 All assertions made by Ramanujan in his 'unpublished' manuscript on the partition and tau functions [1] of the format (3) are false.

Proof. Let $q$ be a prime from Table 1. Assume (3) holds true for $r>1+\delta$, with $\delta$ as in the last column of Table 1 . Then the second order coefficient equals $\delta$, which does not match the value of $C_{2}(t)$ given in Table 1 . For the function $\lambda$ from Section 6 we have $C_{2}(l)=0.2325 \cdots-\frac{1}{2} \log 3 \neq 0.5$.

\section{On a 1928 paper of Geraldine Stanley}

The purpose of Stanley's paper [17] is to show that two assertions due to Ramanujan are false. The first assertion was already mentioned in the introduction. Stanley's analysis of this case contains, unfortunately, several misprints and errors, which are corrected in 16].

The second assertion concerns the $5 \mid \tau(n)$ case. In one of the footnotes we read: "In discussing this question I have used a manuscript of Prof. Hardy, who at one time intended to complete Ramanujan's work". Hardy made some headway with this and then apparently later asked Stanley to fill in the further details. The purpose of this section is to correct Geraldine Stanley's analysis of this case and point out typo's. With respect to the analysis of the first assertion Shanks 16, p. 75] has written: "In fact, there are several errors, and these nullify the proof that Ramanujan's second term is wrong". Indeed, we will see that the same wording applies to her analysis of the $5 \mid \tau(n)$ case. For information on Geraldine Stanley, the reader is referred to [11].

In the definition of $\psi_{1}(s)$, for $p_{2}^{-s}$ read $p_{1}^{-s}$. For $p_{2}^{-s}$ read $p_{1}^{-5 s}$. A few lines down read $(1, i,-i,-1)$ instead of $(1, i,-i, 1)$. For $\left(1-5^{-s}\right)^{5 / 4}$ in the formula for $h(s) \mathrm{read}\left(1-5^{-s}\right)^{3 / 4}$. In the denominator of $h(s)$, the factor $\left(1-p_{3}^{-4 s}\right)^{3 / 4}$ has to be added in the denominator (the so corrected $h(s)$ is thus invariant under 
permuting $p_{2}$ and $p_{3}$ ). In the formula for $A$ at the bottom of $\mathrm{p}$. 236, the factor $(4 / 5)^{3 / 2}$ has to be changed to $4 / 5$. This formula shows that the omission of the factor $\left(1-p_{3}^{-4 s}\right)^{3 / 4}$ in the formula for $h(s)$ was not a mathematical mistake. Also the formula for $a_{1} / A$ given at p. 237 shows that this factor was in the original formula for $h(s)$. The exponent $5 / 4$ instead of $3 / 4$ is consistenly worked with in the remainder of the paper though. This leads then to $(4 / 5)^{3 / 2}$ instead of $4 / 5$ in the formula for $A$ and to $5(\log 5) / 16$ instead of $3(\log 5) / 16$ in the formula for $a_{1} / A$.

From the numerical point of view the formula for $A$ is quite awkward since the values $L_{j}(1), 2 \leq j \leq 4$ have been expanded as Euler products and have been regrouped. (On not doing this a numerically more convenient expression for $A$ can be obtained, cf. Section 4 .) Thus we have to read

$$
\prod\left(\frac{1-p_{4}^{-1}}{\left(1-p^{-1}\right)^{1 / 4}}\right) \text { as } \lim _{x \rightarrow \infty} \frac{\prod_{p_{4} \leq x}\left(1-p_{4}^{-1}\right)}{\prod_{p \leq x}\left(1-p^{-1}\right)^{1 / 4}} .
$$

Indeed, by the Mertens' theorem for arithmetic progressions [23] this limit exists.

The factor $1 /(s+1)^{3 / 4}$ at the middle of p. 237 has to be replaced by $1 /(s-1)^{3 / 4}$. The formula for $b_{1}$ at p. 237 is off by a minus sign (cf. [21, pp. 720-723]). It should read $b_{1}=-a_{1} \Gamma\left(\frac{5}{4}\right) / \pi \sqrt{2}$. Consequently the term $\left(a_{1}-A\right)$ in the first formula for $T(x)$ becomes $\left(-a_{1}-A\right)$ and in the formula for $a_{1} / A, a_{1} / A$ has to be replaced by $-a_{1} / A$. This sign error nullifies the last two sentences of the paper. (Note that apart from the sign error, $5(\log 5) / 16$ has to be replaced by $3(\log 5) / 16$ and $L_{4}(1)$ by $4 L_{4}(1)$.)

\section{References}

[1] B.C. Berndt and K. Ono, Ramanujan's unpublished manuscript on the partition and tau functions with proofs and commentary, The Andrews Festschrift (Maratea, 1998), (Eds.) D. Foata and G.N. Han, 2001, 39-110.

[2] B.C. Berndt and R.A. Rankin, Ramanujan. Letters and commentary, History of Mathematics 9, AMS, London, 1995.

[3] D.A. Cox, Primes of the form $x^{2}+n y^{2}$. Fermat, class field theory and complex multiplication, Wiley, New York, 1989.

[4] K. Dilcher, Generalized Euler constants for arithmetical progressions, Math. Comp. 59 (1999), 259-282.

[5] H.M. Edwards, Fermat's Last Theorem, Graduate Texts in Mathematics 50, Springer-Verlag, New York, 1977.

[6] G.H. Hardy, Ramanujan: twelve lectures on subjects suggested by his life and work, Chelsea Publishing Company, New York, 1959.

[7] J. Knopfmacher, Generalized Euler constants, Proc. Edinburgh Math. Soc. 21 (1978), 25-32. 
[8] E. Landau, Über die zu einem algebraischen zahlkörper gehörige Zetafunktion und die Ausdehnung der Tschebyschefschen Primzahlentheorie auf das Problem der Verteilung der Primideale, J. Reine Angew. Math. 125 (1902), 64-188.

[9] P. Moree, Chebyshev's bias for composite numbers with restricted prime divisors, arXiv:math.NT/0112100, to appear in Math. Comp..

[10] P. Moree and J. Cazaran, On a claim of Ramanujan in his first letter to Hardy, Exposition. Math. 17 (1999), 289-311.

[11] R. A. Rankin, Obituary: Gertrude Katherine Stanley, Bull. London Math. Soc. 14 (1982), 554-555.

[12] R. A. Rankin, Ramanujan's manuscripts and notebooks, Bull. London Math. Soc. 14 (1982), 81-97.

[13] J.B. Rosser and L. Schoenfeld, Approximate formulas for some functions of prime numbers, Illinois Journal Math. 6 (1962), 64-94.

[14] J.-P. Serre, Divisibilité de certaines fonctions arithmétiques, Enseignement Math. 22 (1976), 227-260.

[15] J.-P. Serre, Quelques applications du théorème de densité de Chebotarev, Publ. Math. I. H. E. S. 54 (1981), 123-201.

[16] D. Shanks, The second-order term in the asymptotic expansion of $B(x)$, Math. Comp. 18 (1964), 75-86.

[17] G.K. Stanley, Two assertions made by Ramanujan, Journal London Math. Soc. 3 (1928), 232-237 (1928), Corrigenda, ibid. 4 (1929), 32.

[18] H.P.F. Swinnerton-Dyer, Congruence properties of $\tau(n)$, in Ramanujan revisited (Urbana-Champaign, Ill., 1987), 289-311, Academic Press, Boston, MA, 1988.

[19] L.C. Washington, Introduction to cyclotomic fields, Graduate Texts in Mathematics 83. Springer-Verlag, New York, 1982.

[20] G.N. Watson, Über Ramanujansche Kongruenzeigenschaften der Zerfällungsanzahlen. I, Math. Z. 39 (1935), 712-731.

[21] G.N. Watson, Ramanujans Vermutung über Zerfällungsanzahlen, J. Reine Angew. Math. 179 (1938), 97-128.

[22] G.N. Watson, A table of Ramanujan's function $\tau(n)$, Proc. London Math. Soc. 51 (1949), 1-13.

[23] K.S. Williams, Mertens' theorem for arithmetic progressions, J. Number Theory 6 (1974), 353-359.

[24] J.R. Wilton, Congruence properties of Ramanujan's function $\tau(n)$, Proc. London Math. Soc. 31 (1930), 1-10. 